\title{
Delayed bronchobiliary fistula following radiofrequency ablation in a patient with hepatocellular carcinoma: A case report and lesson regarding treatment
}

\author{
ZHAOLIN ZENG, MINGYUE CAI, WENSOU HUANG, JINGJUN HUANG, XIUZHEN CHEN, \\ HONG SHAN and KANGSHUN ZHU
}

Department of Radiology, The Third Affiliated Hospital of Sun Yat-sen University, Guangzhou, Guangdong 510630, P.R. China

Received January 19, 2015; Accepted February 24, 2016

DOI: $10.3892 / 01.2016 .4366$

\begin{abstract}
Bronchobiliary fistula (BBF) is a rare complication of radiofrequency ablation (RFA) of hepatocellular carcinoma. The rupture of a biloma following RFA may result in the development of BBF, with their early detection and timely management important in the prevention of BBF. The current study presents a case of BBF, which developed at 17 months after radiofrequency ablation (RFA), due to biloma rupture in a patient with hepatocellular carcinoma. Despite the percutaneous drainage of the biloma following BBF, the persistent fever did not resolve due to biliary infection. Finally, an extensive surgical intervention was performed. The magnetic resonance imaging (MRI) scans that had been performed following RFA were reviewed, and it was found that the biloma and increased bile leakage had presented prior to biloma rupture. For that reason, it is advised that patients who present with biloma following RFA should receive regular follow-up MRI scans. Biloma enlargement could be a predictor for the development of BBF; therefore, timely drainage of an enlarging biloma may be able to prevent this complication.
\end{abstract}

\section{Introduction}

Hepatocellular carcinoma (HCC) is reportedly the fifth most common type of cancer and third most common cause of tumor-related mortality worldwide, with an annual incidence of 500,000-1,000,000 (1). Radiofrequency ablation (RFA) is now widely used to treat HCC and hepatic metastasis, as it is minimally invasive, effective and relatively safe. Several clinical studies have demonstrated that RFA may achieve an overall survival rate similar to that of surgical resection in

Correspondence to: Professor Kangshun Zhu, Department of Radiology, The Third Affiliated Hospital of Sun Yat-sen University, 600 Tianhe Road, Guangzhou, Guangdong 510630, P.R. China E-mail: zhksh010@163.com

Key words: bronchobiliary fistula, complications, radiofrequency ablation, hepatocellular carcinoma patients with small $\mathrm{HCC}(2,3)$; however, due to the growing number of patients undergoing the procedure and the increasing number of lesions manifesting in precarious locations, such as the subcapsular region, hepatic dome, peri-hilum area, and areas close to the bile duct and intestinal tract, a number of post-RFA complications have been reported (3-7). Common complications include subcapsular hematoma, hepatic abscess, biloma, pneumothorax and pleural effusion. Rare complications, such as diaphragmatic necrosis and abscesso-colonic and biliopleural fistula have also been reported $(1,8)$.

The present study reports a case of delayed bronchobiliary fistula (BBF), caused by the rupture of a biloma as a rare complication of RFA in a patient with HCC. The biloma and $\mathrm{BBF}$ were resolved by percutaneous biloma drainage and an extensive surgical intervention, including hepatolobectomy, choledocholithotomy and T-tube drainage. Written informed consent was obtained from the patient for publication of the present study and accompanying images.

\section{Case report}

A 57-year-old male patient with chronic hepatitis B (HB), who was suspected to have HCC located in segment VIII following detection of a lesion by Doppler Ultrasound (GE Voluson 730 Expert; GE Healthcare Bio-Sciences, Pittsburgh, PA, USA) during a routine examination, was admitted to The Third Affiliated Hospital of Sun Yat-sen University (Guangzhou, China) on 21 September, 2010. An MRI scan (Signa Excite 1.5T TwinSpeed; GE Healthcare Bio-Sciences) revealed a tumor in segment VIII, which measured 44x34 mm and was in close proximity to the right and middle hepatic veins and right anterior portal vein branch (Fig. 1). The laboratory examinations revealed an $\alpha$-fetoprotein level of $10.27 \mathrm{ng} / \mathrm{ml}$ (normal range, $\leq 20 \mathrm{ng} / \mathrm{ml}$ ) and $\mathrm{HB}$ virus-DNA quantification results of $7.56 \times 10^{3} \mathrm{IU} / \mathrm{ml}$ (normal range, $<100 \mathrm{IU} / \mathrm{ml}$ ). Hematological and biochemical test results for complete blood cell count, prothrombin time, aspartate aminotransferase (AST), alanine aminotransferase (ALT), total bilirubin (TBIL), serum albumin and creatinine, were all normal. AST was measured as $18 / \mathrm{U} / 1$ (normal range, 15-40 U/1), ALT as $20 \mathrm{U} / 1$ (normal range, 3-35 U/1) and TBIL as $12.4 \mu \mathrm{mol} / 1$ (normal range, $4-23.9 \mu \mathrm{mol} / \mathrm{l}$ ). The patient also had a history of type 2 

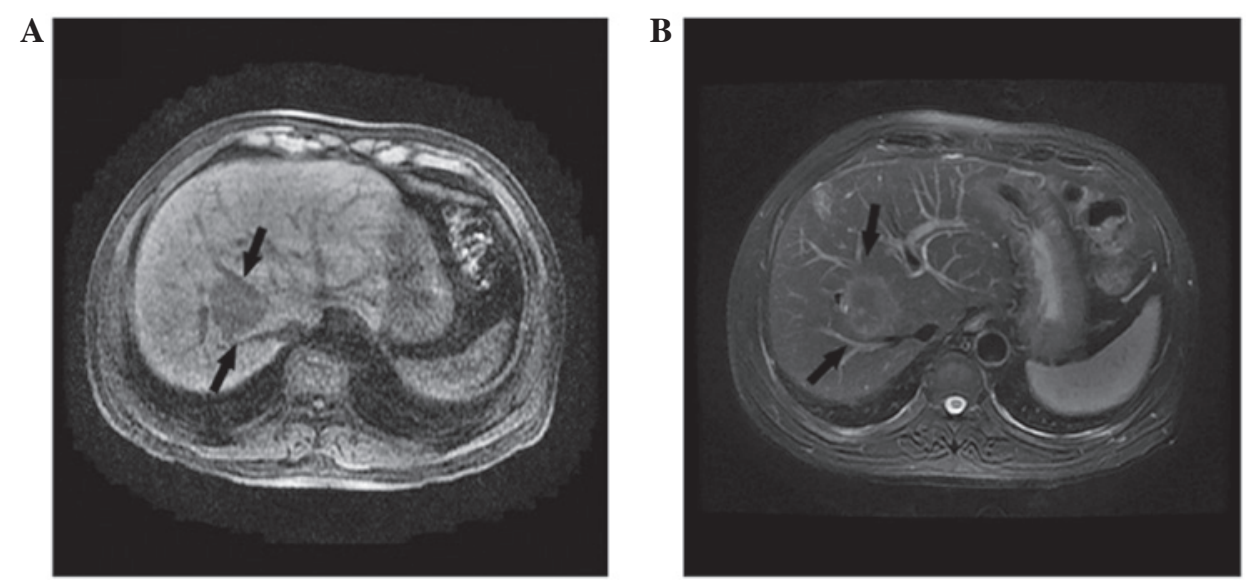

Figure 1. Magnetic resonance imaging scans prior to radiofrequency ablation showing a 44x34-mm tumor in segment VIII with (A) hypointensity on T1-weighted images and (B) hyperintensity on T2-weighted images (arrows).

diabetes mellitus, chronic coronary heart disease and cerebral infarction; therefore, surgical resection was not considered.

A combined therapy of transcatheter arterial chemoembolization (TACE) and percutaneous RFA was adopted. TACE was performed by superselective catheterization of the tumor-feeding artery. An emulsion of $6 \mathrm{ml}$ lipiodol (Guerbet, Aulnay-sous-Bois, France) and $30 \mathrm{mg}$ doxorubicin hydrochloride (Pfizer, Inc., Wuxi, China) was infused into the artery, which was followed by an embolization of gelatin sponge particles (Hangzhou AILIKANG Medicine Technology Co. Ltd., Hangzhou, China). At 11 days after TACE, the RFA procedure was performed with ultrasonography guidance under general anesthesia using a $10-\mathrm{cm}$ long cool-tip electrode with a $2-\mathrm{cm}$ active tip and a 200-W RF generator (Radionics, Inc., Burlington, MA, USA). A total of 8 overlapping ablations, each lasting $12 \mathrm{~min}$, were performed.

Abdominal MRI performed 1 month after RFA revealed an ablation zone of $75 \times 54 \mathrm{~mm}$ reaching the right diaphragm, and a small amount of right pleural effusion (Fig. 2). At 17 months after RFA, the patient was re-admitted to the hospital after presenting with fever and an irritating cough lasting for 1 week. The laboratory findings were as follows: AST, $64 \mathrm{U} / 1$; ALT, $20 \mathrm{U} / \mathrm{l}$; TBIL, $57.8 \mu \mathrm{mol} / 1$. The light-yellow sputum was confirmed by laboratory biochemical testing to have bile components. Computed tomography (CT) scans (AQUILION ONE ${ }^{\mathrm{TM}}$; Toshiba Medical Systems, Otawara, Japan) of the chest and abdomen revealed a biloma at the ablation zone, which ruptured into the thoracic and abdominal cavities. In addition, pneumonia and atelectasis were observed in the right lower lung lobe, and a small right pleural effusion, as well as biliary sludge and small biliary calculi, were detected in the dilated bile duct (Fig. 3).

Ultrasonography-guided percutaneous transhepatic biloma drainage was performed using an 8-French pigtail catheter. The fluid that was aspirated from the biloma during the procedure contained yellow bile and brown biliary sludge, in which Escherichia coli was identified following bacterial culture. BBF was further suggested by an injection of $2 \mathrm{ml}$ methylene blue solution into the biloma through the drainage catheter, following which the methylene blue solution was expectorated from the mouth. X-ray angiography performed through the drainage catheter showed that the biloma communicated with the biliary system, with multiple small biliary calculi found in the dilated bile ducts.

One month after the biloma drainage, the cough had subsided but the fever persisted. Chest and abdominal CT scans demonstrated the improvement of pneumonia and right pleural effusion; however, the biliary sludge and small biliary calculi endured in the dilated bile duct. Despite repeated drainage and treatment with antibiotics for 1 month, the fever did not resolve. The cause of the fever was considered to be a biliary system infection that had not been controlled by the percutaneous biloma drainage, and an extensive surgical intervention including hepatolobectomy, choledocholithotomy and T-tube drainage was performed. Intraoperatively, an active leak was detected at the start of the right hepatic ducts, which allowed for communication between the biloma and the biliary system. In addition, a large volume of dark brown biliary sludge and calculi, which had led to biliary obstruction, dilatation and infection were detected. The biloma and BBF were successfully treated and the patient was discharged 40 days after the surgery. On 10 March, 2014, tumor recurrence was detected in segment II by MRI. RFA was successfully performed on March 14, 2014; A total of 2 overlapping ablations, each lasting $12 \mathrm{~min}$, were performed. To date, no further tumor recurrence has been detected during follow-up examinations, in addition to no recurrence of biloma or BBF.

\section{Discussion}

$\mathrm{BBF}$ is a rare disease that was first reported by Peacock in 1850 (9). BBF is an abnormal communication between the bile duct and the bronchial tree, and can arise from hydatid disease, trauma, bile duct obstruction, tumor invasion and iatrogenic injuries caused by procedures such as TACE and RFA $(10,11)$. The complication rate of RFA for HCC is $0.6-10 \%(4,12)$; however, only sporadic cases of BBF as a complication of RFA have been reported (13-15). To the best of our knowledge, this is the first reported case of delayed BBF caused by biloma rupture following RFA.

The most specific symptom of BBF is expectoration of yellow, bile-tinged sputum (13). Biochemical examination of the sputum may identify BBF based on the presence of bile components. CT scan is used as the first-line imaging technique. 

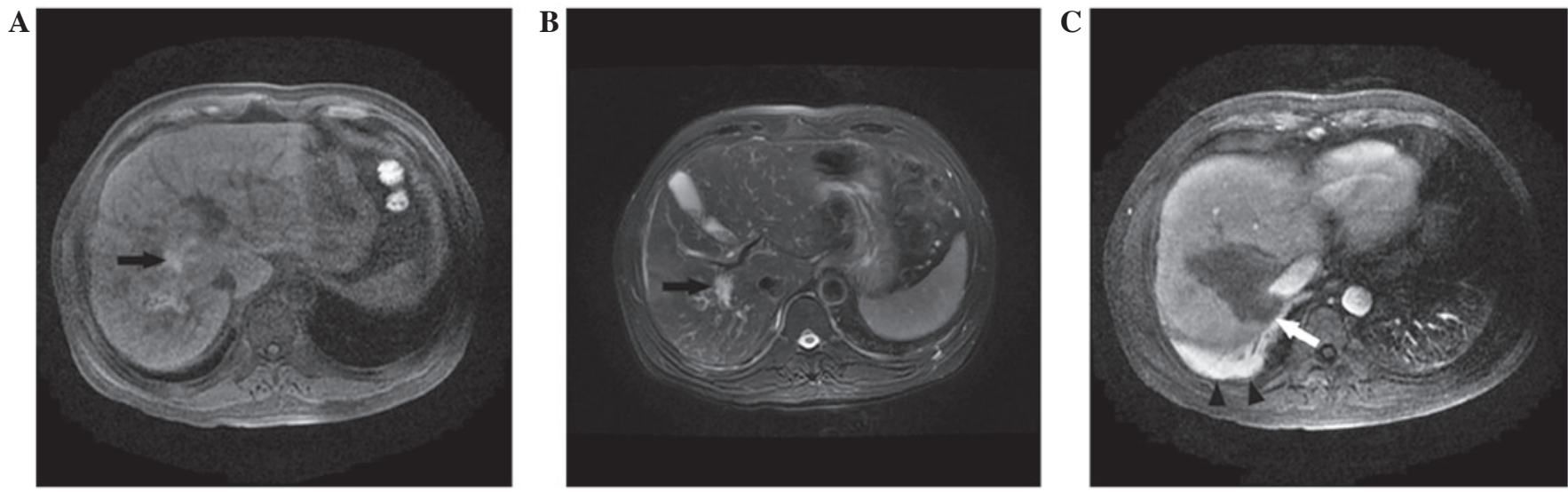

Figure 2. MRI scans 1 month after radiofrequency ablation. The (A) T1-weighted and (B) T2-weighted transverse images with a high signal at the ablation zone (black arrows), which was later confirmed as bile leakage. (C) An ablation zone of $75 \times 54 \mathrm{~mm}$ was shown to reach the right diaphragm on portal venous phase contrast-enhanced MRI scans (white arrow), with a small amount of pleural effusion (black arrowheads). MRI, magnetic resonance imaging.
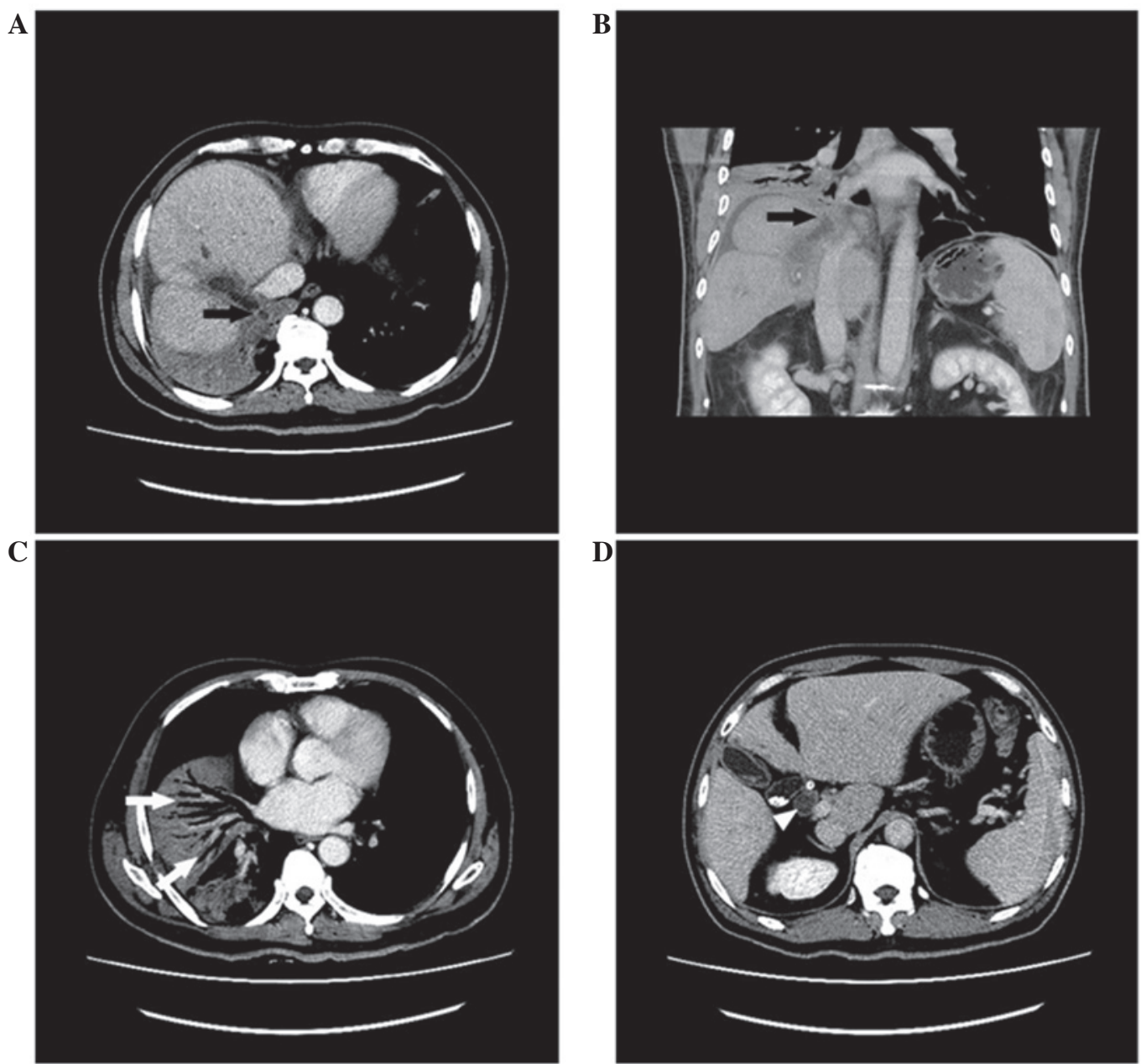

Figure 3. Chest and abdominal computed tomography scans obtained at 17 months after radiofrequency ablation. (A and B) The biloma ruptured into the pleural and abdominal cavity via the injured diaphragm (black arrows), with (C) pneumonia and atelectasis observed in the right lower lung lobe (white arrow) and (D) biliary sludge and small biliary calculi detected in the dilated bile duct (white arrowhead).

Despite the fact that it may not directly display the fistula tract, it can reveal indirect findings, such as pneumonia, subphrenic fluid collection, pleural effusion, liver abscess, biloma and bile duct calculi $(8,11,14)$. Contrast-enhanced magnetic resonance cholangiopancreatography is a non-invasive technique that may be used to diagnose BBF and can directly detect the fistula tract (16). Invasive examinations, such as percutaneous transhepatic cholangiography or endoscopic retrograde cholangiopancreatography, can reveal the abnormal fistula tract between the biliary system and the bronchial tree, which is considered to be the most direct evidence to suggest $\operatorname{BBF}(8,12)$. In the present case, the patient's sputum was confirmed to 


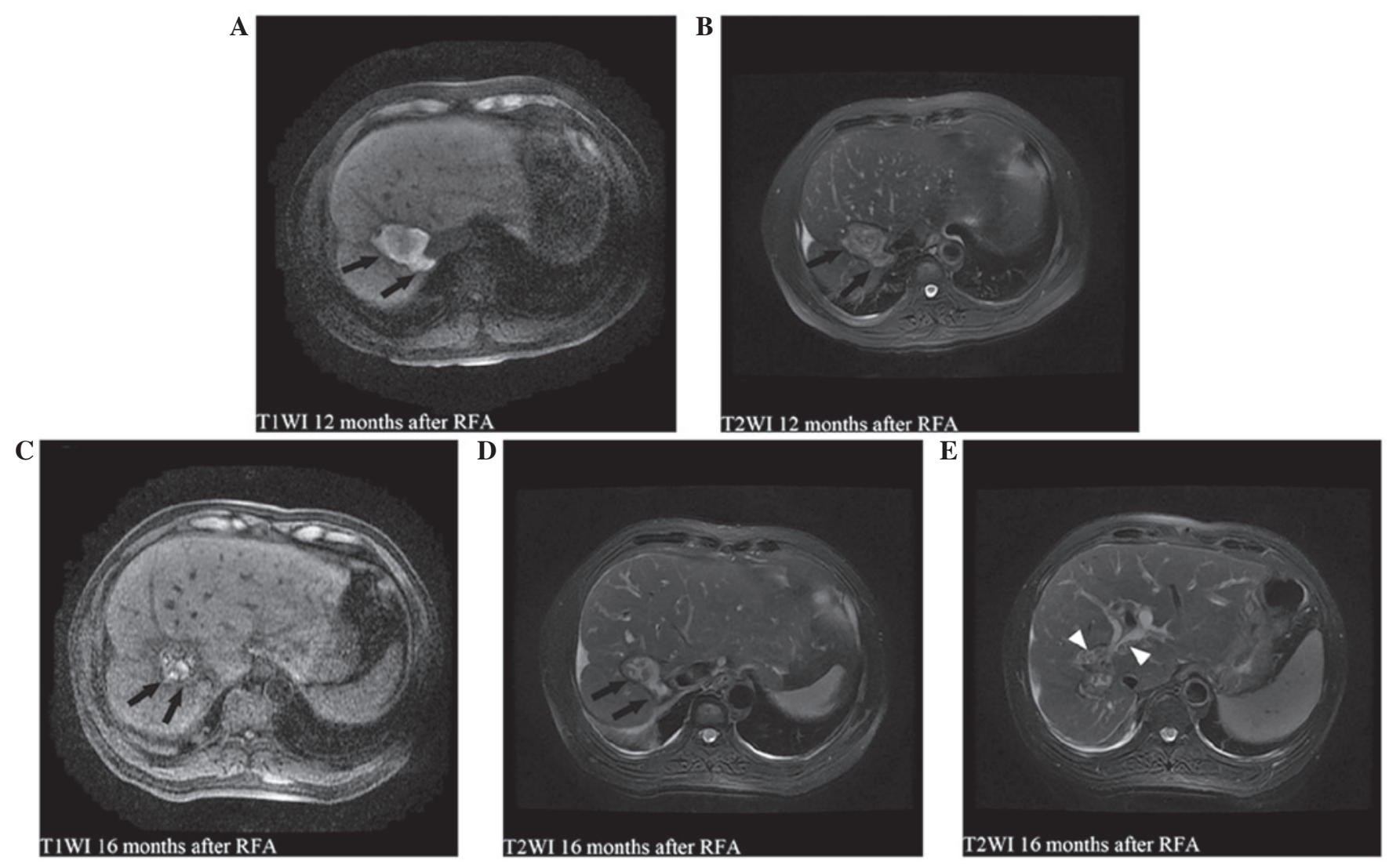

Figure 4. MRI scans at 12 and 16 months after RFA showing (A-E) a biloma at the ablation zone (black arrows) and an increase in bile leakage compared with the MRI scans performed at 1 month after RFA (Fig. 2). (C-E) The biliary sludge and stones in the biloma and dilated bile duct were also detected on MRI scans at 16 months after RFA (white arrowheads). MRI, magnetic resonance imaging; RFA, radiofrequency ablation; T1WI, T1-weighted imaging; T2WI, T2-weighted imaging.

contain bile components by biochemical examination, and methylene blue solution was injected into the biloma through the drainage catheter and was later expectorated from the mouth. Both examinations are able to confirm the diagnosis of BBF.

In the present case, the delayed BBF following RFA was most likely a joint result of various factors, such as the formation of the biloma, the damage to the diaphragm abutting the biloma, biliary infection and bile duct obstruction.

The damage to the diaphragm and the formation of a biloma may be predominantly attributed to the thermal effects of RFA on the diaphragm and bile duct. A large tumor, close to the hilum or hepatic dome, has been found to be a predisposing factor of $\operatorname{BBF}$ following RFA $(8,11)$. In the present case, a liver tumor measuring $44 \times 34 \mathrm{~mm}$, which was located near the right hepatic bile duct and dome, was treated with RFA, and the ablation zone was found to have reached a size of $75 \times 54 \mathrm{~mm}$ on MRI at 1 month after RFA. The ablation zone margin was found to reach the diaphragm and a small amount of right pleural effusion was observed on the MRI scan (Fig. 2), suggesting the possibility of diaphragmatic injury. Although no active biliary fistula leak was directly detected by MRI scan at 1 month after RFA, bile leakage was observed (Fig. 2). The hepatectomy performed later also confirmed that the biliary fistula originated from the right hepatic bile duct, which directly communicated with the biloma. In addition, the bile duct obstruction, which had been caused by a large volume of biliary sludge and stones, could have contributed to the rising pressure of the biloma and its later rupture, as the increase in bile leakage, biliary sludge and stones and bile duct dilation (Fig. 4) were detected on the follow-up MRI scan prior to the rupture of the biloma. The existence of a biloma might have also caused the repeated biliary infection, which was responsible for the fever that persisted even after percutaneous biloma drainage was performed; therefore, in the present case, various pathological conditions could have simultaneously contributed to the formation of a delayed BBF.

The primary treatment for BBF is relief of the bile duct obstruction and drainage of the subphrenic or hepatic abscesses and biloma. Less invasive treatment procedures include endoscopic or percutaneous biliary drainage or percutaneous biloma drainage $(8,13)$. Most BBFs can be resolved by drainage and biliary tract decompression; however, certain BBFs persist, requiring surgical intervention (9). The surgical approach often adopted by clinicians involves thoracicoabdominal exploration, including hepatolobectomy, resection of the fistula tract, pulmonary lobectomy and cholangioenterostomy, alone or as a combination of various surgical approaches. In the present case, after the patient underwent percutaneous biloma drainage, the pneumonia and subphrenic and pleural effusions were nearly resolved; however, the persistent fever was not resolved, which could have been due to insufficient drainage of the biloma by percutaneous drainage alone. Bile duct obstruction, which was caused by a large volume of biliary sludge and stones, contributed to the insufficient drainage, which, in turn, caused repeated biliary 
infection and persistent fever. An extensive surgical intervention was required in order to resect the biloma and remove the biliary sludge and stones.

Had the biloma and its enlargement been identified early on, during the follow-up MRI scan, and the percutaneous biloma drainage been performed in a timely manner, the development of BBF and subsequent surgical intervention could have been prevented. In fact, the biloma was present 1-16 months after RFA, before the patient presented with fever and coughing up of bile-tinged sputum. The biloma was retrospectively observed on a review of the MRI scan performed 1 month after RFA (Fig. 2). In addition, on the follow-up MRI scans at 3, 6, 12 and 16 months after RFA, biloma enlargement, bile duct obstruction, caused by a large volume of biliary sludge and stones, and bile duct dilation were detected (Fig. 4). The bile duct obstruction led to an increase in the bile leakage and pressure of the biloma, prior to the rupture of the biloma. These results were supported by the hepatolobectomy that was later performed. The findings of the present study suggested that, when biloma enlargement is observed on follow-up MRI scans, timely percutaneous biloma drainage should be performed to prevent its rupture and the development of BBF.

In conclusion, for patients with large tumors close to the hepatic dome and hilum, RFA should be cautiously performed, in order to prevent thermal injury to the diaphragm and bile duct. In addition, regular follow-up MRI scans after RFA are required for the early detection of biloma, and percutaneous biloma drainage should be performed as soon as an enlarging biloma is detected by MRI scan, for the prevention of biloma rupture and BBF development.

\section{Acknowledgments}

This study was supported by the National Natural Science Foundation of China (grant no. 81371655) and the Science and Technology Planning Project of Guangdong Province, China (grant no. 2010B031600211).

\section{References}

1. Kim JY, Kwon YH, Lee SJ, Jang SY, Yang HM, Jeon SW and Kweon YO: Abscesso-colonic fistula following radiofrequency ablation therapy for hepatocellular carcinoma: A case successfully treated with histoacryl embolization. Korean J Gastroenterol 58: 270-274, 2011 (In Korean).
2. Peng ZW, Lin XJ, Zhang YJ, Liang HH, Guo RP, Shi M and Chen MS: Radiofrequency ablation versus hepatic resection for the treatment of hepatocellular carcinoma $2 \mathrm{~cm}$ or smaller: A retrospective comparative study. Radiology 262: 1022-1033, 2012.

3. Solbiati L, Ahmed M, Cova L, Ierace T, Brioschi M and Goldberg SN: Small liver colorectal metastases treated with percutaneous radiofrequency ablation: Local response rate and long-term survival with up to 10 -year follow-up. Radiology 265 : 958-968, 2012

4. Minami Y and Kudo M: Radiofrequency ablation of hepatocellular carcinoma: Current status. World J Radiol 2: 417-424, 2010.

5. Teratani T, Yoshida H, Shiina S, Obi S, Sato S, Tateishi R, Mine N Kondo Y, Kawabe T and Omata M: Radiofrequency ablation for hepatocellular carcinoma in so-called high-risk locations. Hepatology 43: 1101-1108, 2006.

6. Chang IS, Rhim H, Kim SH, Kim YS, Choi D, Park Y and Lim HK: Biloma formation after radiofrequency ablation of hepatocellular carcinoma: Incidence, imaging feature, and clinical significance. AJR Am J Roentgenol 195: 1131-1136, 2010.

7. Livraghi T, Solbiati L, Meloni MF, Gazelle GS, Halpern EF and Goldberg SN: Treatment of focal liver tumors with percutaneous radiofrequency ablation: Complications encountered in a multicenter study. Radiology 226: 441-451, 2003.

8. Pende V, Marchese M, Mutignani M, Polinari U, Allegri C, Greco R and Costamagna G: Endoscopic management of biliopleural fistula and biloma after percutaneous radiofrequency ablation of liver metastasis. Gastrointest Endosc 66: 616-618, 2007.

9. Peacock TB: Case in which hydatids were expectorated and one of suppuration of hydatid cyst of the liver communicating with the lungs. Edinburgh Med Surg J 74: 33-46, 1850.

10. Liao GQ, Wang H, Zhu GY, Zhu KB, Lv FX and Tai S: Management of acquired bronchobliary fistula: A systematic literature review of 68 cases published in 30 years. World J Gastroentrol 17: 3842-3849, 2011.

11. Yoon DH, Shim JH, Lee WJ, Kim PN, Shin JH and Kim KM: Percutaneous management of a bronchobiliary fistula after radiofrequency ablation in a patient with hepatocellular carcinoma. Korean J Radiol 10: 411-415, 2009.

12. Kong WT, Zhang WW, Qiu YD, Zhou T, Qiu JL, Zhang W and Ding YT: Major complications after radiofrequency ablation for liver tumors: Analysis of 255 patients. World J Gastroenterol 15: 2651-2656, 2009.

13. Kim YS, Rhim H, Sung JH, Kim SK, Kim Y, Koh BH, Cho OK and Kwon SJ: Bronchobiliary fistula after radiofrequency thermal ablation of hepatic tumor. J Vasc Interv Radiol 16: 407-410, 2005

14. Tran T, Hampel H, Qureshi WA and Shaib Y: Successful endoscopic management of bronchobiliary fistula due to radiofrequency ablation. Dig Dis Sci 52: 3178-3180, 2007.

15. Kim DH, Choi DW, Choi SH, Heo JS, Jeong J and Rhu J: Surgical treatment of bronchobiliary fistula due to radiofrequency ablation for recurrent hepatocellular carcinoma. Korean J Hepatobiliary Pancreat Surg 17: 135-138, 2013.

16. Karabulut N, Cakmak V and Kiter G: Confident diagnosis of bronchobiliary fistula using contrast-enhanced magnetic resonance cholangiography. Korean J Radiol 11: 493-496, 2010. 